\title{
GEOMETRIC MODELLING OF OCTAGONAL LAMP POLES
}

\author{
Ting On Chan and Derek D. Lichti \\ Department of Geomatics Engineering, University of Calgary \\ 2500 University Dr NW, Calgary, Alberta, T2N1N4 Canada \\ (ting.chan, ddlichti)@ucalgary.ca
}

Commission V, WG V/3

KEY WORDS: LiDAR, Octagonal Pole, Geometric Modelling, Polygon

\begin{abstract}
:
Lamp poles are one of the most abundant highway and community components in modern cities. Their supporting parts are primarily tapered octagonal cones specifically designed for wind resistance. The geometry and the positions of the lamp poles are important information for various applications. For example, they are important to monitoring deformation of aged lamp poles, maintaining an efficient highway GIS system, and also facilitating possible feature-based calibration of mobile LiDAR systems. In this paper, we present a novel geometric model for octagonal lamp poles. The model consists of seven parameters in which a rotation about the $\mathrm{z}$ axis is included, and points are constrained by the trigonometric property of $2 \mathrm{D}$ octagons after applying the rotations. For the geometric fitting of the lamp pole point cloud captured by a terrestrial LiDAR, accurate initial parameter values are essential. They can be estimated by first fitting the points to a circular cone model and this is followed by some basic point cloud processing techniques. The model was verified by fitting both simulated and real data. The real data includes several lamp pole point clouds captured by: (1) Faro Focus 3D and (2) Velodyne HDL-32E. The fitting results using the proposed model are promising, and up to $2.9 \mathrm{~mm}$ improvement in fitting accuracy was realized for the real lamp pole point clouds compared to using the conventional circular cone model. The overall result suggests that the proposed model is appropriate and rigorous.
\end{abstract}

\section{INTRODUCTION}

Roadside pole-like objects such as lamp poles are of interest for many applications such as three-dimensional (3D) city modelling and for geographic information systems (GIS). Therefore they are often the segmentation targets from large point clouds (Cabo et al., 2014; Halawany and Lichti, 2013; Pu et al., 2011; Yokoyama et al. 2011; Lehtomäki et al., 2010). However, the geometric properties of pole-like objects such as lamp poles are not modelled and estimated preciously in most cases.

If pole-like objects, particularly octagonal lamp poles, are accurately modelled geometrically, they could be treated as calibration references for terrestrial LiDAR and mobile mapping systems (Chan and Lichti, 2012, 2013; Chan et al., 2013) due to their abundance in most road scenes. In fact, developing an accurate geometric model for such pole-like objects can also be beneficial to many real-life problems such as pole reconstruction for 3D city modelling (Buhur et al., 2009), pole deformation monitoring (Chang et al., 2009) and quality control of pole manufacturing (Lindskog et al., 2012). Since most tall lamp poles usually have octagonal base and can be modelled as octagonal cones/pyramids, a rigorous geometric model of an octagonal cone is proposed in this paper.

For in situ calibration of mobile LiDAR, a rigorous 3D geometric model which equates all the $\mathrm{x}, \mathrm{y}$ and $\mathrm{z}$ coordinates for the octagonal cone is essential and is therefore of this paper. Some research (e.g., Milenkovic, 1993) has addressed the problem of polygon modelling but the models are mostly in two-dimensional (2D) and the models and their associated constraints often consist of inequalities. Chicurel-Uziel (2004) proposed a generalized 3D model for polygonal prisms without inequality but the $\mathrm{x}$ and $\mathrm{y}$ coordinates are modelled and estimated separately using two equations and they cannot be readily expressed in terms of the other. Also, no inclinations of the prism were considered in its model.

\section{THE PROPOSED MODEL FOR OCTAGONAL CONE}

\subsection{The Proposed Model}

The fundamental concept of the proposed model is that by breaking the cone into many horizontal 2D octagonal layers, all eight sides of the octagon can be treated as the same one side by applying the transformation as a regular octagon is rotational symmetric. The side is then constrained with a simple trigonometric equation augmented with a half of the fixed interior angle of the octagon. The proposed model is consists of seven parameters: the cone centre $\left(X_{\mathrm{c}}, Y_{\mathrm{c}}\right)$, the rotations about the $\mathrm{X}$-axis $(\Omega)$, Y-axis $(\Phi)$ and $\mathrm{Z}$-axis $(\Psi)$, the octagonal radius $\left(R_{0}\right)$ at $Z=0$ and also the cone gradient factor $(k)$ as depicted in Figure 1. Some of the parameters are inherited from the conventional cylindrical model which has five degrees of freedom (Rabbani et al., 2007). The octagonal cone has one additional rotation parameter $(\Psi)$ about the Z-axis on the top of the cylindrical model because an octagon is not isotropic about the Z-axis.

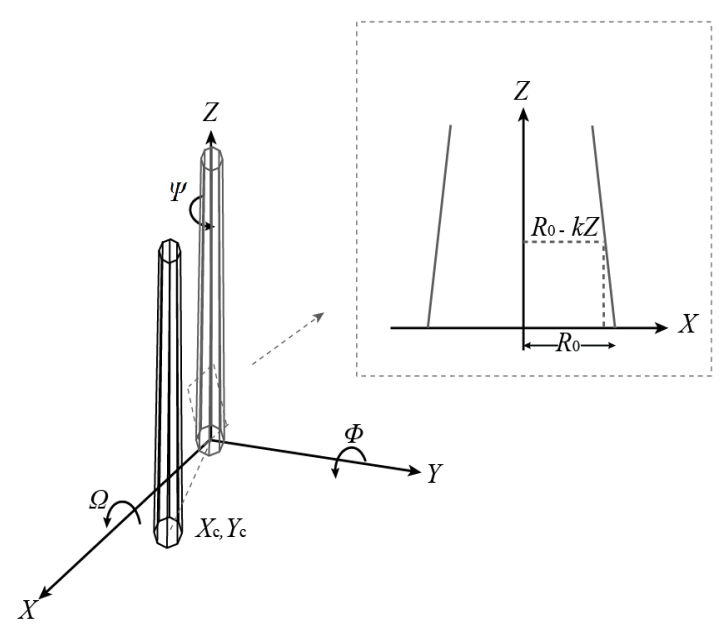

Figure 1. The parameters of the proposed octagonal cone model 
After the cone is reduced to the origin by subtracting the cone centre $\left(X_{\mathrm{c}}, Y_{\mathrm{c}}\right)$, it is rotated about the $X$ - and $Y$ - axes by $\Omega$ and $\Phi$ respectively. It is followed by further rotating the cone about the Z-axis by $\Psi\left(-22.5^{\circ} \leq \Psi \leq 22.5^{\circ}\right)$ as illustrated in Figure 2a (only showing the cross section at $\mathrm{Z}=0$ ) to its nominal position (Figure 2b).
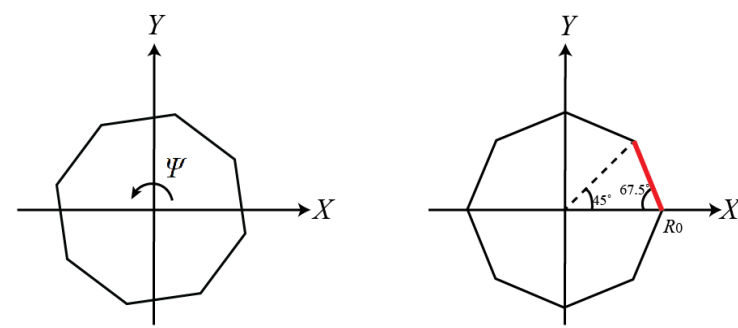

Figure 2. Rotation about the $Z$-axis with $\Psi$ (2a, left); The nominal position and the trigonometric constraint in the first octant ( $2 \mathrm{~b}$, right)

With the octagonal cone transformed to the nominal position, points lying on the octagonal side of the first octant (red in Figure 2 b) satisfy the following trigonometric constraint

$$
\left(R_{0}-X^{\prime}\right) \tan \left(67.5^{\circ}\right)=Y^{\prime}
$$

where $R_{0}$ is defined as the octagonal radius which is the distance between the centre and a vertex of the octagon on the $X Y$-plane after the cone is transformed to be perpendicular to the $X Y$-plane.

Every point lying on the cone can be classified as belonging to one of the eight octants (Figure 3) depending on the point's position and $q$ is defined as the octant number.

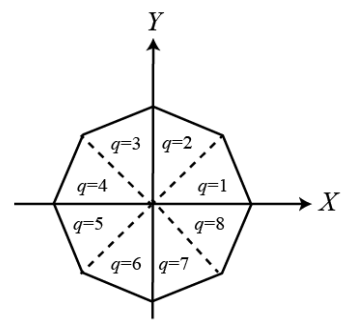

Figure 3. The octant number $(q)$ for the model

By computing $q$, points lying on other octants (the $2^{\text {nd }}, 3^{\text {rd }}, \ldots$, $8^{\text {th }}$ octant) can also be constrained by Equation (1) after rotating the points about $\mathrm{Z}$-axis by the following angle

$$
(q-1) 45^{\circ}+\Psi
$$

and $q$ can be calculated as follows

$$
q=\left\lceil\frac{\Theta}{45^{\circ}}\right\rceil
$$

where $\Theta\left(0^{\circ}<\Theta \leq 360^{\circ}\right)$ is the angle from the $X$-axis on the $X Y$ plane. The angle $\Theta$ can be readily computed from $\hat{X}$ and $\hat{Y}$ coordinates of the point after the cone is transformed to the nominal position by

$$
\left(\begin{array}{c}
\hat{X} \\
\hat{Y} \\
\hat{Z}
\end{array}\right)=\mathbf{R}_{\mathbf{3}}(\Psi) \mathbf{R}_{2}(\Phi) \mathbf{R}_{1}(\Omega)\left(\begin{array}{c}
X-X_{\mathrm{c}} \\
Y-Y_{\mathrm{c}} \\
Z
\end{array}\right)
$$

On the other hand, the octagonal radius decreases with increasing height (Z). The gradient factor, $k$, governs the radius decrement by subtracting a small portion of $\mathrm{Z}$ from $R_{0}$. Therefore, the overall geometric model for the octagonal cone is

$$
f(\vec{x}, \vec{l})=\left[\left(R_{0}-k Z^{\prime}\right)-X^{\prime}\right] \tan \left(67.5^{\circ}\right)-Y^{\prime}=0
$$

where

$$
\left(\begin{array}{c}
X^{\prime} \\
Y^{\prime} \\
Z^{\prime}
\end{array}\right)=\mathbf{R}_{3}\left((q-1) \cdot 45^{\circ}+\Psi\right) \mathbf{R}_{2}(\Phi) \mathbf{R}_{1}(\Omega)\left(\begin{array}{c}
X-X_{\mathrm{c}} \\
Y-Y_{\mathrm{c}} \\
Z
\end{array}\right)
$$

Note that Equations (5) and (6) can be generalized for other polygonal cones such as hexagonal cone, which is given by

$$
f(\vec{x}, \vec{l})=\left[\left(R_{0}-k Z^{\prime}\right)-X^{\prime}\right] \tan \left(\left(1-\frac{2}{n}\right) \cdot 90^{\circ}\right)-Y^{\prime}=0
$$

where

$$
\left(\begin{array}{c}
X^{\prime} \\
Y^{\prime} \\
Z^{\prime}
\end{array}\right)=\mathbf{R}_{3}\left((q-1) \cdot \frac{360^{\circ}}{n}+\Psi\right) \mathbf{R}_{2}(\Phi) \mathbf{R}_{1}(\Omega)\left(\begin{array}{c}
X-X_{\mathrm{c}} \\
Y-Y_{\mathrm{c}} \\
Z
\end{array}\right)
$$

and $n$ is the number of sides for the corresponding polygon.

\subsection{Initial Parameter Estimation}

The initial values of all the parameters (except $\Psi$ ) of the proposed model can be estimated using least-squares fitting with the circular cone model

$$
f(\vec{x}, \vec{l})=X^{\prime 2}+Y^{\prime 2}-\left(R_{0}-k Z^{\prime}\right)^{2}=0
$$

where

$$
\left(\begin{array}{c}
X^{\prime} \\
Y^{\prime} \\
Z^{\prime}
\end{array}\right)=\mathbf{R}_{2}(\Phi) \mathbf{R}_{1}(\Omega)\left(\begin{array}{c}
X-X_{\mathrm{c}} \\
Y-Y_{\mathrm{c}} \\
Z
\end{array}\right)
$$

The initial values for the above model can be all set to zero except for $R_{0}$, for which only a rough estimate is required (e.g. based on empirical knowledge).

After obtaining the six initial values, the cone can be reduced to the origin and rotated to be perpendicular to the $X Y$-plane. Then the initial value of $\Psi$ can be found by first isolating a thin octagonal layer around $\mathrm{Z}=0(\sim 1 \mathrm{~cm}$ thick). This is followed by projection of the points on the layer onto a $2 \mathrm{D}$ image (Figure 4a). Therefore, the octagonal sides can be readily detected using region growing or the Hough Transform, and the vertices of the 
octagon are thus found. $\Psi$ is the smallest angle between the $X$ axis and the vertex which is the closest to the $X$-axis. Figure $4 \mathrm{~b}$ shows the projected 2D image of cone in the nominal position after rotating by $\Psi$ for $12^{\circ}$.
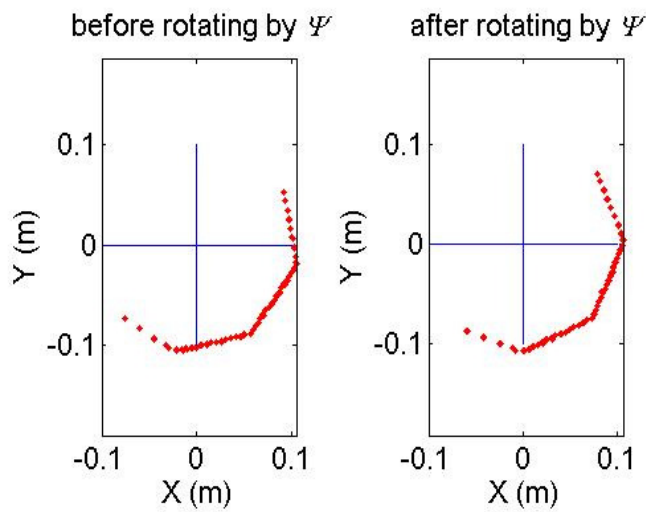

Figure 4. Projected 2D image from the point cloud for calculating approximate value of $\Psi$ (4a, left): before rotating by $\Psi ;(4 \mathrm{~b}$, right): after rotating by $\Psi$

\section{EXPERIMENT}

A full errorless vertical octagonal lamp pole was simulated with the realistic parameters shown in Table 1 . The height of the simulated octagonal pole is $11 \mathrm{~m}$ and it consists of 140864 points. Figure 5 shows the simulated pole and its projected cross-section on the $X Y$-plane Least-squares fitting of the data to the proposed model (Equation 5) was performed.
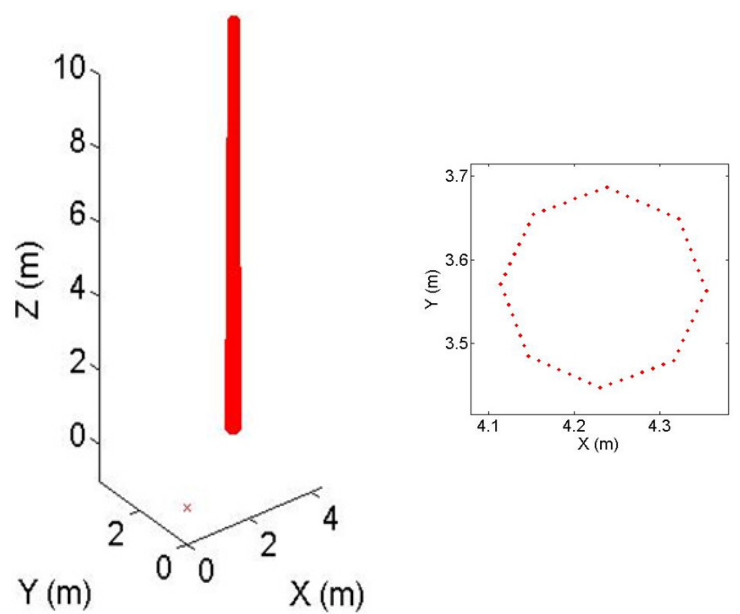

Figure 5 . The simulated octagonal lamp pole (5a, left) and its projected cross section on the $X Y$-plane ( $5 \mathrm{~b}$, right)

Table 1. The parameter values for the simulated octagonal pole

\begin{tabular}{|c|c|}
\hline & Simulated Value \\
\hline$X_{\mathrm{c}}(\mathrm{m})$ & 4.2340 \\
\hline$Y_{\mathrm{c}}(\mathrm{m})$ & 3.5670 \\
\hline$\Omega(\mathrm{rad})$ & -0.0050 \\
\hline$\Phi(\mathrm{rad})$ & 0.0060 \\
\hline$\Psi(\mathrm{rad})$ & -0.0349 \\
\hline$k$ & 0.0050 \\
\hline$R_{0}(\mathrm{~m})$ & 0.1200 \\
\hline
\end{tabular}

Five real octagonal lamp poles of two different sizes were captured with the Faro Focus 3D (Figure 6) and the Velodyne HDL-32E (Figure 7) on the University of Calgary campus. The scanner was placed approximately $4 \mathrm{~m}$ in average away from the poles. Least-squares fitting using the octagonal cone model (Equation 5) was performed with the lamp pole point clouds (the vertical part) captured by Faro Focus 3D (Lamp Poles 1 3 ) and the HDL-32E (Lamp Poles 4 and 5). Only 4 sides of the pole were captured for each scanner position. The Velodyne point clouds contain time varying errors (Glennie and Lichti, 2011; Chan et al., 2013) and thus the least-squares convergence thresholds have to be tuned based on the severity of the noise. The details of the poles and fitting input are summarized in Table 2. The point clouds of Poles 1 and 4 are plotted in Figures 8 and 9 respectively.

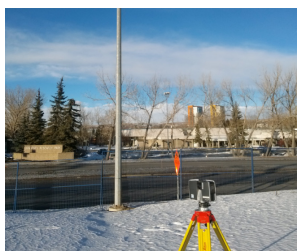

Figure 6. A lamp pole was captured with the Faro Focus 3D on the University of Calgary Campus

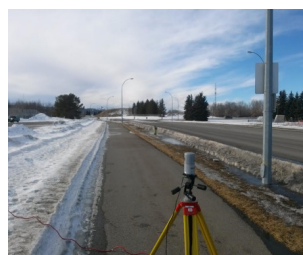

Figure 7. A lamp pole was captured with the Velodyne HDL-32E on the University of Calgary Campus
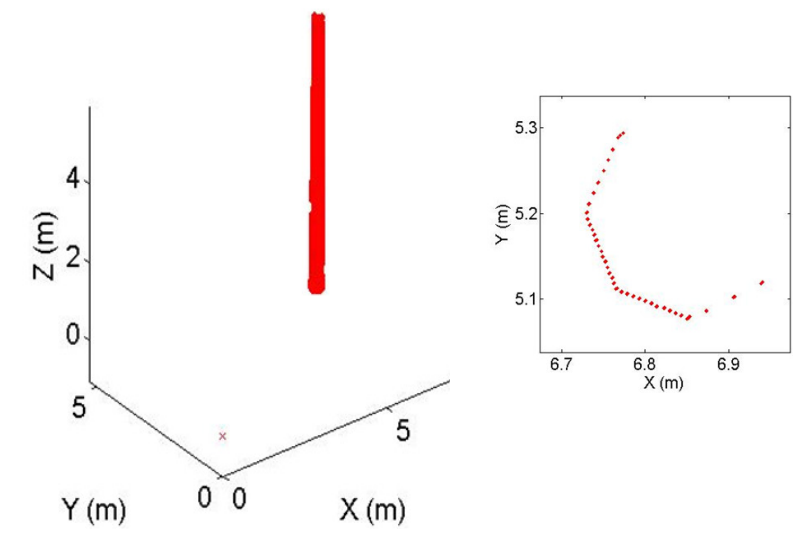

Figure 8. The point cloud of Pole 1captured by Faro Focus 3D (8a, left) and its projected cross section on the $X Y$-plane ( $8 \mathrm{~b}$, right)

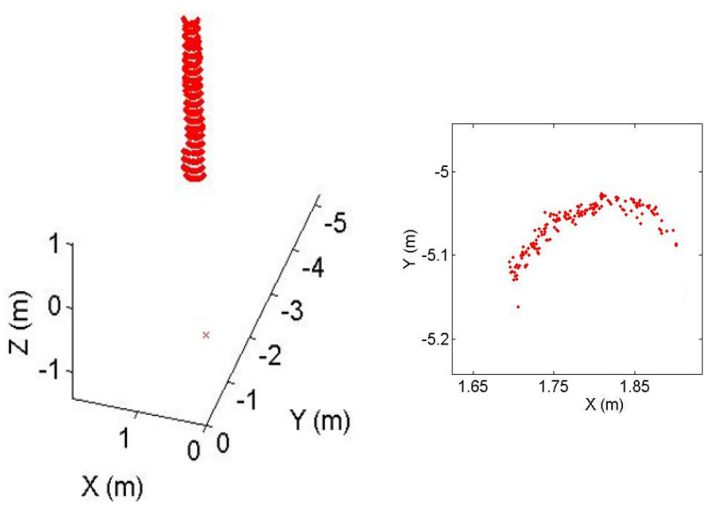

Figure 9. The point cloud of Pole 1captured by Velodyne HDL-32E (9a, left) and its projected cross section on the $X Y$-plane (9b, right) 
Table 2. Summary of the real data fitting

\begin{tabular}{|c|c|c|c|c|c|}
\hline Pole & Scanner & $\begin{array}{l}\text { No of } \\
\text { points }\end{array}$ & $\begin{array}{l}\text { Approx. } \\
\text { Length } \\
\text { (m) }\end{array}$ & $\begin{array}{l}\text { Approx } \\
R_{0}(\mathrm{~m})\end{array}$ & $\begin{array}{lll}\text { Observation } \\
\sigma_{\mathrm{x} /} & \sigma_{\mathrm{y} /} & \sigma_{\mathrm{z}} \\
(\mathrm{m}) & & \end{array}$ \\
\hline 1 & \multirow{3}{*}{$\begin{array}{c}\text { Faro } \\
\text { Focus 3D }\end{array}$} & 30867 & 7 & 0.12 & \multirow{3}{*}{0.003} \\
\hline 2 & & 75386 & 7 & 0.12 & \\
\hline 3 & & 60146 & 12 & 0.13 & \\
\hline 4 & \multirow{2}{*}{$\begin{array}{l}\text { Velodyne } \\
\text { HDL-32E }\end{array}$} & 3327 & 7 & 0.12 & \multirow{2}{*}{0.010} \\
\hline 5 & & 4059 & 7 & 0.12 & \\
\hline
\end{tabular}

\section{RESULTS}

\subsection{Model Fitting Results with the Simulated Data}

Table 3 shows the estimated parameter values and their precision from the least-squares fitting of the simulated pole. All the parameters were accurately recovered with high precision as seen in Tables 1 and 3. Also, no high parameter correlation was found from the correlation matrix (absolute value) tabulated in Table 4.

Table 3. Estimates of octagonal cone model fitting with the simulated data

\begin{tabular}{|c|c|c|}
\hline & Est. Value & Std. Dev. \\
\hline$X_{\mathrm{c}}(\mathrm{m})$ & 4.2340 & $1.05 \mathrm{E}-04$ \\
\hline$Y_{\mathrm{c}}(\mathrm{m})$ & 3.5670 & $1.05 \mathrm{E}-04$ \\
\hline$\Omega(\mathrm{rad})$ & -0.0050 & $2.18 \mathrm{E}-05$ \\
\hline$\Phi(\mathrm{rad})$ & 0.0060 & $2.18 \mathrm{E}-05$ \\
\hline$\Psi(\mathrm{rad})$ & -0.0349 & $1.82 \mathrm{E}-03$ \\
\hline$k$ & 0.0050 & $1.70 \mathrm{E}-05$ \\
\hline$R_{0}(\mathrm{~m})$ & 0.1200 & $8.66 \mathrm{E}-05$ \\
\hline
\end{tabular}

Table 4. Correlation matrix of octagonal cone model fitting with the simulated data

\begin{tabular}{|c|c|c|c|c|c|c|c|}
\hline & $X_{\mathrm{c}}$ & $Y_{\mathrm{c}}$ & $\Omega$ & $\Phi$ & $\Psi$ & $k$ & $R_{0}$ \\
\hline$X_{\mathrm{c}}$ & 1.00 & 0.00 & 0.00 & 0.76 & 0.00 & 0.00 & 0.00 \\
\hline$Y_{\mathrm{c}}$ & & 1.00 & 0.76 & 0.00 & 0.01 & 0.00 & 0.00 \\
\hline$\Omega$ & & & 1.00 & 0.00 & 0.00 & 0.00 & 0.00 \\
\hline$\Phi$ & & & & 1.00 & 0.00 & 0.00 & 0.00 \\
\hline$\Psi$ & & & & & 1.00 & 0.17 & 0.38 \\
\hline$k$ & & & & & & 1.00 & 0.76 \\
\hline$R_{0}$ & & & & & & & 1.00 \\
\hline
\end{tabular}

\subsection{Model Fitting Results with the Real Data}

\subsubsection{Faro Focus 3D}

The mean estimated results from the least-squares fitting using the octagonal cone model for Poles 1-3 captured by the Faro Focus 3D are shown in Table 5. It is shown that the precisions of the parameters are all high and a reasonable estimated variance factor $\left(\hat{\sigma}_{0}^{2}\right)$ is obtained (the observation standard deviations for the adjustment are listed in Table 2).

Unlike the eight-sided simulated pole, some high correlations between the parameters were found from the correlation matrix (Table 6) which shows the mean absolute values of the correlation coefficients for Poles 1 - 3. Only 4 sides (half of the pole) for each pole were observed from a scanner position and this leads to rather poor geometry for the adjustment so that $R_{0}$ becomes more coupled to either $X_{\mathrm{c}}$ or $Y_{\mathrm{c}}$, or both depending the relative position between the pole and the scanner. Similarly, $k$ is more coupled with the inclinations of the pole due to lack of observations of the complete pole. Fitting the same data to a circular cone also incurs these high correlation problems.
Table 5. Mean estimates of octagonal cone model fitting with the real data (Pole 1 - 3) captured by the Faro Focus 3D

\begin{tabular}{|c|c|c|}
\hline & Est. Value & Std. Dev. \\
\hline$X_{\mathrm{c}}(\mathrm{m})$ & -0.5057 & $6.29 \mathrm{E}-05$ \\
\hline$Y_{\mathrm{c}}(\mathrm{m})$ & 3.9494 & $5.47 \mathrm{E}-05$ \\
\hline$\Omega(\mathrm{rad})$. & 0.0030 & $1.73 \mathrm{E}-05$ \\
\hline$\Phi(\mathrm{rad})$. & -0.0022 & $1.99 \mathrm{E}-05$ \\
\hline$\Psi(\mathrm{rad})$. & -0.0391 & $6.00 \mathrm{E}-04$ \\
\hline$k$ & 0.0062 & $2.11 \mathrm{E}-05$ \\
\hline$R_{0}(\mathrm{~m})$ & 0.1160 & $6.71 \mathrm{E}-05$ \\
\hline$\hat{\sigma}_{0}^{2}$ & \multicolumn{2}{|c|}{0.82} \\
\hline
\end{tabular}

Table 6. Mean correlation matrix of octagonal cone model fitting with the real data (Pole $1-3$ ) captured by the Faro Focus 3D

\begin{tabular}{|c|c|c|c|c|c|c|c|}
\hline & $X_{\mathrm{c}}$ & $Y_{\mathrm{c}}$ & $\Omega$ & $\Phi$ & $\Psi$ & $k$ & $R_{0}$ \\
\hline$X_{\mathrm{c}}$ & 1.00 & 0.66 & 0.49 & 0.74 & 0.04 & 0.67 & 0.90 \\
\hline$Y_{\mathrm{c}}$ & & 1.00 & 0.73 & 0.50 & 0.05 & 0.62 & 0.83 \\
\hline$\Omega$ & & & 1.00 & 0.67 & 0.01 & 0.83 & 0.61 \\
\hline$\Phi$ & & & & 1.00 & 0.00 & 0.90 & 0.67 \\
\hline$\Psi$ & & & & & 1.00 & 0.01 & 0.02 \\
\hline$k$ & & & & & & 1.00 & 0.75 \\
\hline$R_{0}$ & & & & & & & 1.00 \\
\hline
\end{tabular}

On the other hand, the mean precision of Poles $1-3$ from the lamp-pole fitting of the octagonal and circular cone models is compared (Table 7). It can be seen that the octagonal model delivered higher precision for all the parameters. Furthermore, the root mean square (RMS) values of the octagonal model fitting residuals are also lower in all the three dimensions. Up to $2.9 \mathrm{~mm}$ mean RMS discrepancy between two models' residuals was realized as shown in Table 8. As a result, the proposed model is a more accurate and thus more appropriate for modelling the octagonal lamp pole point clouds.

Table 7. Comparison of the mean estimated standard deviations of the octagonal and circular cone models fitting with the real data (Poles 1 3) captured by the Faro Focus 3D

\begin{tabular}{|c|c|c|}
\hline & $\begin{array}{c}\text { Octagonal Cone } \\
\text { Model Fitting }\end{array}$ & $\begin{array}{c}\text { Circular Cone } \\
\text { Model Fitting }\end{array}$ \\
\hline & Mean Std. Dev. & Mean Std. Dev. \\
\hline$X_{\mathrm{c}}(\mathrm{m})$ & $6.29 \mathrm{E}-05$ & $8.44 \mathrm{E}-05$ \\
\hline$Y_{\mathrm{c}}(\mathrm{m})$ & $5.47 \mathrm{E}-05$ & $7.32 \mathrm{E}-05$ \\
\hline$\Omega(\mathrm{rad})$ & $1.74 \mathrm{E}-05$ & $2.30 \mathrm{E}-05$ \\
\hline$\Phi(\mathrm{rad})$ & $1.98 \mathrm{E}-05$ & $2.65 \mathrm{E}-05$ \\
\hline$\Psi(\mathrm{rad})$ & $6.00 \mathrm{E}-04$ & - \\
\hline$k$ & $2.11 \mathrm{E}-05$ & $2.60 \mathrm{E}-05$ \\
\hline$R_{0}(\mathrm{~m})$ & $6.71 \mathrm{E}-05$ & $8.31 \mathrm{E}-05$ \\
\hline
\end{tabular}

Table 8. Comparison of the mean RMS of the residuals of the octagonal and circular cone models fitting with the real data (Poles 1 - 3) captured

\begin{tabular}{|c|c|c|c|}
\hline \multirow{2}{*}{} & \multicolumn{3}{|c|}{ by the Faro Focus 3D } \\
\cline { 2 - 4 } & $\mathrm{RMS}_{\mathrm{x}}(\mathrm{mm})$ & $\mathrm{RMS}_{\mathrm{y}}(\mathrm{mm})$ & $\mathrm{RMS}_{\mathrm{z}}(\mathrm{mm})$ \\
\hline $\begin{array}{c}\text { Octagonal Cone } \\
\text { Model Fitting }\end{array}$ & 1.71 & 1.65 & 0.02 \\
\hline $\begin{array}{c}\text { Circular Cone } \\
\text { Model Fitting }\end{array}$ & 4.60 & 4.30 & 0.05 \\
\hline
\end{tabular}

\subsubsection{Velodyne HDL-32E}

For the Velodyne point cloud for Poles 4 - 5, the fitting with the octagonal cone model also gives realistic estimates (except $k$ ) with high precision and also a small estimated variance factor as shown in Table 9. The estimated $k$ is large because the differences between systematic errors adherent to the points captured by different laser heads are high and this causes the 
whole point cloud deviated extensively from the conic structure in which the radius is reduced by a small fixed ratio $(k)$ when the vertical position increase. Furthermore, the parameter correlations are fairly low except the $\Omega-k$ and $Y_{\mathrm{c}}-R_{0}$ correlations as seen in Table 10. A similar conclusion from Section 4.2.1 can be drawn for explaining that.

Table 9. Mean estimates of octagonal cone model fitting with the real data (Pole 4 - 5) captured by the Velodyne HDL-32E

\begin{tabular}{|c|c|c|}
\hline & Est. Value & Std. Dev. \\
\hline$X_{\mathrm{c}}(\mathrm{m})$ & 2.1412 & $4.51 \mathrm{E}-04$ \\
\hline$Y_{\mathrm{c}}(\mathrm{m})$ & -1.5914 & $5.52 \mathrm{E}-04$ \\
\hline$\Omega(\mathrm{rad})$ & 0.0029 & $7.91 \mathrm{E}-04$ \\
\hline$\Phi(\mathrm{rad})$ & 0.0155 & $6.49 \mathrm{E}-04$ \\
\hline$\Psi(\mathrm{rad})$ & -0.0466 & $7.47 \mathrm{E}-03$ \\
\hline$k$ & 0.0158 & $8.20 \mathrm{E}-04$ \\
\hline$R_{0}(\mathrm{~m})$ & 0.1059 & $5.70 \mathrm{E}-04$ \\
\hline$\hat{\sigma}_{0}^{2}$ & \multicolumn{2}{|c|}{0.85} \\
\hline
\end{tabular}

Table 10. Mean correlation matrix of octagonal cone model fitting with the real data (Pole 4 -5) captured by the Velodyne HDL-32E

\begin{tabular}{|c|c|c|c|c|c|c|c|}
\hline & $X_{\mathrm{c}}$ & $Y_{\mathrm{c}}$ & $\Omega$ & $\Phi$ & $\Psi$ & $k$ & $R_{0}$ \\
\hline$X_{\mathrm{c}}$ & 1.00 & 0.49 & 0.17 & 0.39 & 0.13 & 0.25 & 0.69 \\
\hline$Y_{\mathrm{c}}$ & & 1.00 & 0.28 & 0.18 & 0.09 & 0.22 & 0.84 \\
\hline$\Omega$ & & & 1.00 & 0.47 & 0.03 & 0.85 & 0.21 \\
\hline$\Phi$ & & & & 1.00 & 0.03 & 0.66 & 0.27 \\
\hline$\Psi$ & & & & & 1.00 & 0.02 & 0.01 \\
\hline$k$ & & & & & & 1.00 & 0.29 \\
\hline$R_{0}$ & & & & & & & 1.00 \\
\hline
\end{tabular}

Both the simulated and real data fitting results suggest that the proposed model is appropriate and rigorous. In addition, the results also show that the proposed model more accurately models octagonal lamp poles as compared to the conventional circular cone model.

\section{CONCLUSIONS}

This paper presents a novel octagonal cone model for lamp pole point clouds. The model is in implicit form and can be readily implemented with the least-squares fitting. The model was verified with simulated data and also real data of several lamp pole point clouds captured by a panoramic terrestrial LiDAR (the Faro Focus 3D) and a spinning beam LiDAR (the Velodyne HDL-32E). The results suggest that the proposed model is appropriate and rigorous. In addition, the modelling concept developed in this paper can also be readily transferred and applied to other 3D polygonal cone such as hexagonal cone. Future research work is to apply the propose models to sensor/system calibration problems.

\section{ACKNOWLEDGEMENTS}

The work was supported by the Tecterra Inc. and the Werner Graupe International Fellowship in Engineering in Canada.

\section{REFERENCES}

Buhur, S., Ross, L., Büyüksalih,G., Baz, I., 2009. 3D city modelling for planning activities, case Study: Haydarpasa Train Station, Haydarpasa Port and Surrounding Backside Zones, Istanbul. ISPRS Proceedings XXXVIII-1-4-7 W5, (On CDRom)
Cabo, C., Ordoñez, C., García-Cortés, S., \& Martínez, J., 2014. An algorithm for automatic detection of pole-like street furniture objects from mobile laser scanner point clouds. ISPRS Journal of Photogrammetry and Remote Sensing 87, pp. 47-56.

Chan, T. O. and Lichti, D. D., 2012. Cylinder-based selfcalibration of a panoramic terrestrial laser scanner. The International Archives of the Photogrammetry, Remote Sensing and Spatial Information Sciences 39 (Part B5), pp. 169-174.

Chan, T. O. and Lichti, D. D., 2013. Feature-based selfcalibration of Velodyne HDL-32E LiDAR for terrestrial mobile mapping applications. The 8th International Symposium on Mobile Mapping Technology, Tainan, Taiwan, 1st -3rd May, 2013. (On CD-Rom)

Chan, T. O., Lichti, D. D. and Belton D., 2013. Temporal analysis and automatic calibration of the Velodyne HDL-32E LiDAR system. ISPRS Annals - Volume II-5/W2, 2013, pp. 61-66.

Chang, B., Phares B. M., Sarkar P. P., and Wipf, T. J., 2009. Development of a procedure for fatigue design of slender support structures subjected to wind-induced vibration. Transportation Research Record: Journal of the Transportation Research Board 2131, pp. 23-33.

Chicurel-Uziel, E., 2004. Single equation without inequalities to represent a composite curve. Computer Aided Geometric Design 21(1), pp. 23-42.

El-Halawany S. I. and Lichti, D. D., 2013. Detecting road poles from mobile terrestrial laser scanning data. GIScience \& Remote Sensing 50:6, pp. 704-722.

Glennie, C. and Lichti, D.D., 2011. Temporal stability of the Velodyne HDL-64E S2 scanner for high accuracy scanning applications. Remote Sensing 3, pp. 539-553.

Lehtomäki, M., Jaakkola, A., Hyyppä, J., Kukko, A. and Kaartinen, H., 2010. Detection of vertical pole-like objects in a road environment using vehicle-based laser scanning data. Remote Sensing 2, pp.641-664.

Lindskog, E.; Berglund, J.; Vallhagen, J.; Berlin, R.; Johansson, B., 2012. Combining point cloud technologies with discrete event simulation. Simulation Conference (WSC), Proceedings of the 2012 Winter, pp.1-10, 9-12 Dec. 2012 doi: 10.1109/WSC.2012.6465210.

Milenkovic, V., 1993. Robust polygon modelling. Special Issue of Computer-Aided Design on Uncertainties in Geometric Computations 25(9), pp. 546-566.

Rabbani, T., Dijkman, S., van den Heuvel, F. and Vosselman, G., 2007. An integrated approach for modelling and global registration of point clouds. ISPRS Journal of Photogrammetry and Remote Sensing 61 (6), pp. 355-370.

$\mathrm{Pu}, \mathrm{S}$., Rutzinger, M., Vosselman, G. and Oude Elberink, S.J., 2011. Recognizing basic structures from mobile laser scanning data for road inventory studies. ISPRS Journal of Photogrammetry and Remote Sensing 66 (6), pp. 28-39. 
The International Archives of the Photogrammetry, Remote Sensing and Spatial Information Sciences, Volume XL-5, 2014 ISPRS Technical Commission V Symposium, 23 - 25 June 2014, Riva del Garda, Italy

Yokoyama, H., Date, H., Kanai, S., and Takeda, H., 2011. Polelike object recognition from mobile laser scanning data using smoothing and principal component analysis. International Archives of Photogrammetry, Remote Sensing and Spatial Information Sciences, XXXVIII-5/W12, pp. 115-120. 